\title{
Design and Implementation of Online Examination System based on ASP.NET
}

\author{
Yuxiang Hou \\ Nanchang Institute of Science \& Technology, Nanchang,330108, China
}

Key words: Online examination, ASP.NET

\begin{abstract}
With the continuous updating of educational ideas and the continuous development of computer science and technology, the traditional examination methods have been unable to meet the needs of teachers and students. The examination system based on the network emerges as the times require, not only saves a lot of manpower and material resources, but also improves the flexibility of examination.

With the rapid development of the Internet, the construction of campus information has become a new hot spot in the network application. As a part of the construction for campus information, the online examination system is becoming popular. The online examination system based on $\mathrm{B} / \mathrm{S}$ breaks through the limitation of the area and is loved by many teachers and examinees.

The way of online examination has many advantages, such as convenient and flexible, easy to manage and so on, so that information technology can be better integrated into education and teaching, is in line with the trend of the development for education information and is the inevitable trend of education development.
\end{abstract}

\section{Functional Requirements Analysis}

Compared with the traditional examination mode, online examination system based on B/S can dynamically manage various examination information, including automatic generation of various test papers, automatic calculation of test results and so on. At the same time, it also has a high degree of flexibility, wherever the students are, they can visit the online examination system through the browser.

The main goal of the online examination system is to realize the system to send the question and examination automatically. After the examination is over, the system can also read the paper under a certain plan according to the reference answer. To achieve this goal, we need to systematically and effectively manage examination questions, automatically generate examination papers, and retrieve papers on time.

The analysis of the specific functional requirements for the system is as follows:

(1) User login

Student login: enter student ID, password and verification code correctly, log in, and enter the student examination page.

Teacher login: enter the sign, password and verification code correctly, log in, enter the teacher management page, the course information management, examination question bank management and artificial marking examination papers.

Administrator login: enter the user name, password and verification code correctly, log in, and enter the backstage page.

(2)Backstage management

User management: manage student information, teacher information and administrator information, including the operation of adding, modifying, deleting, and querying.

Professional information management: manage student profession, including the operation of profession adding, modifying, deleting, querying.

Examination subject management: according to the profession to divide examination subjects, including the operation of adding, modifying, deleting, querying for professional examination subjects, and set the prescribed examination time, only in the prescribed time test time for the 
examinee to carry out the examination.

Examination paper management: according to the examination subjects to carry out the examination papers, the type of examination paper is divided into two types of objective questions and subjective questions. The objective questions generally include questions such as filling empty, single choice, multiple choice, judgment, and so on. The subjective questions usually include simple answer, noun interpretation, discussion, calculation, programming and other types of questions. By setting the number and type of questions, the corresponding questions are randomly selected from the questions, and examination paper is formed.

Examination questions management: manage various types of test questions, including the operation of adding, modifying, deleting, and inquirying for single choice, multiple choice, filling empty, judgment, simple answer, noun interpretation, discussion, calculation, programming and other questions.

(3)Student examination

Online examination:

The examinee opens the browser to enter the examination page, enters the login verification information and confirms no error, the system randomly extracts the test question, the examinee begins to answer the paper, the system automatically countdown. At the end of the answer, confirm the assignment, finish the examination, or the time has arrived, the examinee has not finished the answer, and the system will force the ending of the answer, and automatically score and archive the examination questions after the completion of the examination.

Result query: check the student's examination result list.

The functional division of the system is shown in Figure 1.

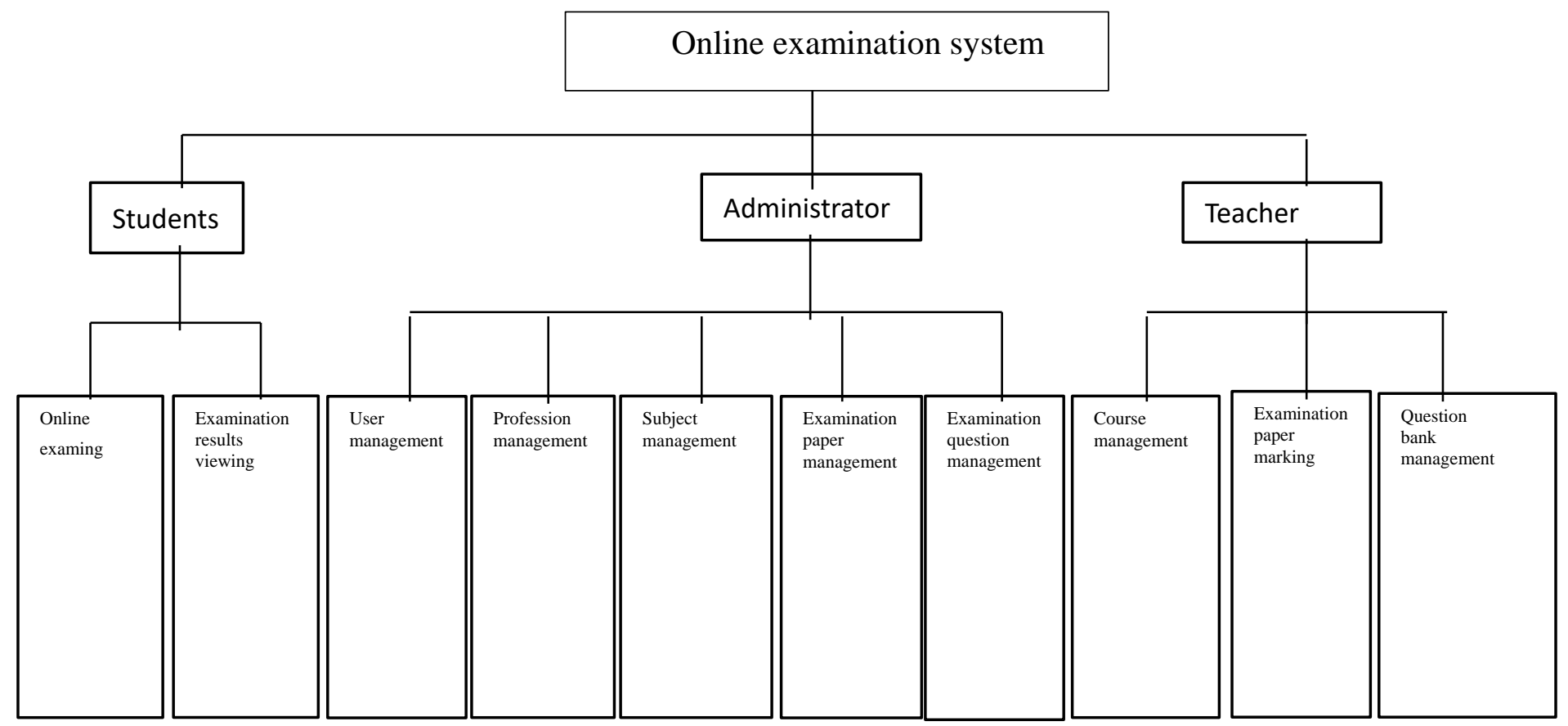

Figure 1 The functional division of the system

\section{System Design}

\subsection{Architecture design}

The online examination system based on ASP.NET is developed by the integrated development environment of Visual Studio 2015, the front page is written with ASP. NET, the backstage program is written in C\# language, and the database uses SQL Server 2008. 


\subsection{Data storage structure design}

The system database server adopts SQL Server 2008, based on C\#.NET platform, data is accessed through ADO.NET. The main data table of the system is as follows:

(1) The administrator information table contains administrator number, administrator name, administrator password and registration time field.

(2) The subject information table contains the subjects number and the subject names field .

(3) Professional information table contains numbering, professional name and creation time.

(4) The score information table contains numbers, student numbers, test paper numbers, scores, and time to answer questions.

(5) The student information table contains student numbers, student names, passwords, sex, registration time, security questions, questions and answers, and majors.

(6) The examination paper information table includes numbering, subject number, examination paper name and examination paper status field.

(7) The detailed information table of the examination paper includes numbering, test paper number, test question type, question number, content and question score field.

(8) The answer information table includes answer number, test number, student number, answer content and topic score.

(9) The result table keeps information about students' related subjects, including achievement number, student number, subject number and total score.

\section{Main Functional Modules of the System}

The main functions of the system designed as follows:

\subsection{Online examination module}

The online examination module is mainly realized students' examination and the results inquirying after the end of the exam. After entering the examination system, the students choose the subject of the exam, get the examination papers, and start the test time. Without submitting the examination paper, you can modify the questions that have been written out. It is not completed until the end of the examination, and the examination paper can be submitted by themselves. After the end of the exam, students can no longer continue to answer, and the main selection of papers is mandatory by the system.

\subsection{Examination question management}

Examination question management is a module for teachers to upload test questions, provides single selection, multiple selection, filling empty, judgment, simple answer, noun interpretation, discussion, calculation, programming, etc., and supports uploading pictures and files, and supports the batch import of test questions. After uploading questions, you can see, modify and delete.

\subsection{Examination paper management}

The main function of the examination paper management module is the organization of the paper, in order to facilitate to give the examination papers, the system provides the function of the test paper template, and defines the composition of the test paper through the template, including the question type, the knowledge point covering, the quantity of the title, the difficulty degree and the fraction distribution. Therefore, the subjectivism of the teacher's manual work can be avoided, especially in the public course. In particular, the students need many sets of test papers, which guarantee the coverage of the knowledge points and the consistency of the difficulty degree to some extent, thus it is beneficial to the principle of fairness and fairness in the examination. In addition, in order to avoid students cheating, we can disrupt each student's test order by random display algorithm.

\subsection{Reading paper management module}

The module contains two modules of marking papers and results analysis. The examination 
papers marking includes automatic reading and manual review, and the automatic review is generally aimed at objective questions, such as filling in the blanks, choosing questions and judging questions, the automatic answer is achieved by comparing the corresponding answers between the student's answer book and the question bank, and manual grading is used to evaluate the subjective questions according to the needs of teachers.

\subsection{User management module}

User management module is mainly used to manage basic information of teachers and students. Both students and teachers, administrators will set up their scope of permission to log in with accounts and passwords to avoid students and teachers to change some of the key information on their own.

\subsection{Subject management module}

The subject management module is mainly about the examination subjects. The administrators can add, delete and change the examination subjects in this module.

\subsection{Professional information management module}

Professional information management module includes professional information querying, professional information adding, professional information deleting, professional information function modifying.

\subsection{Performance management module}

The score management module includes student achievement querying, student achievement adding, student achievement deleting, student's score modifying.

\section{System Running Result}

\subsection{System login interface}

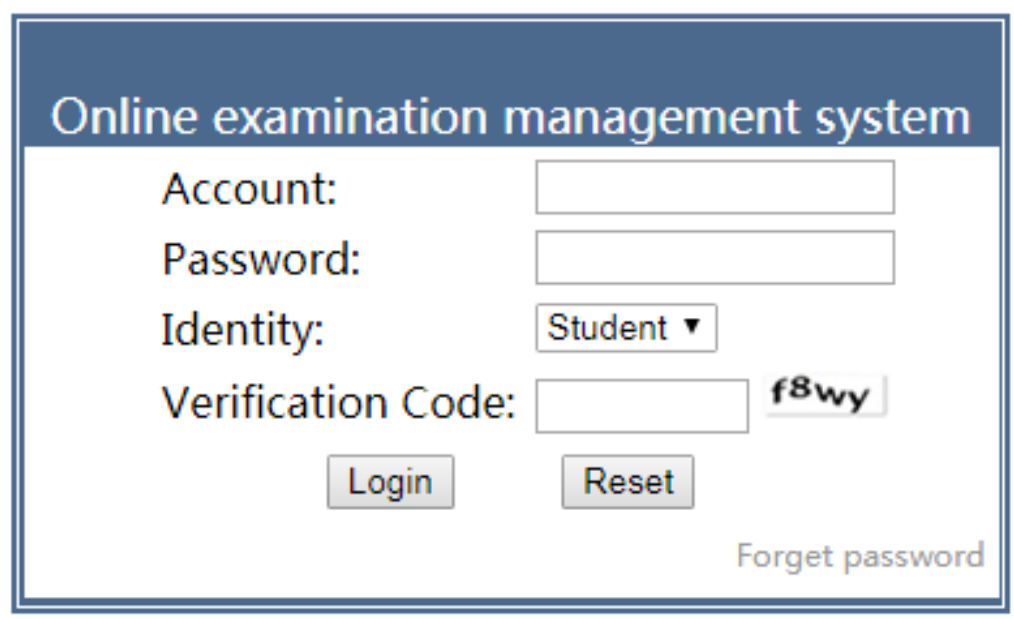

Figure 2 System login interface 


\subsection{Backstage management interface}

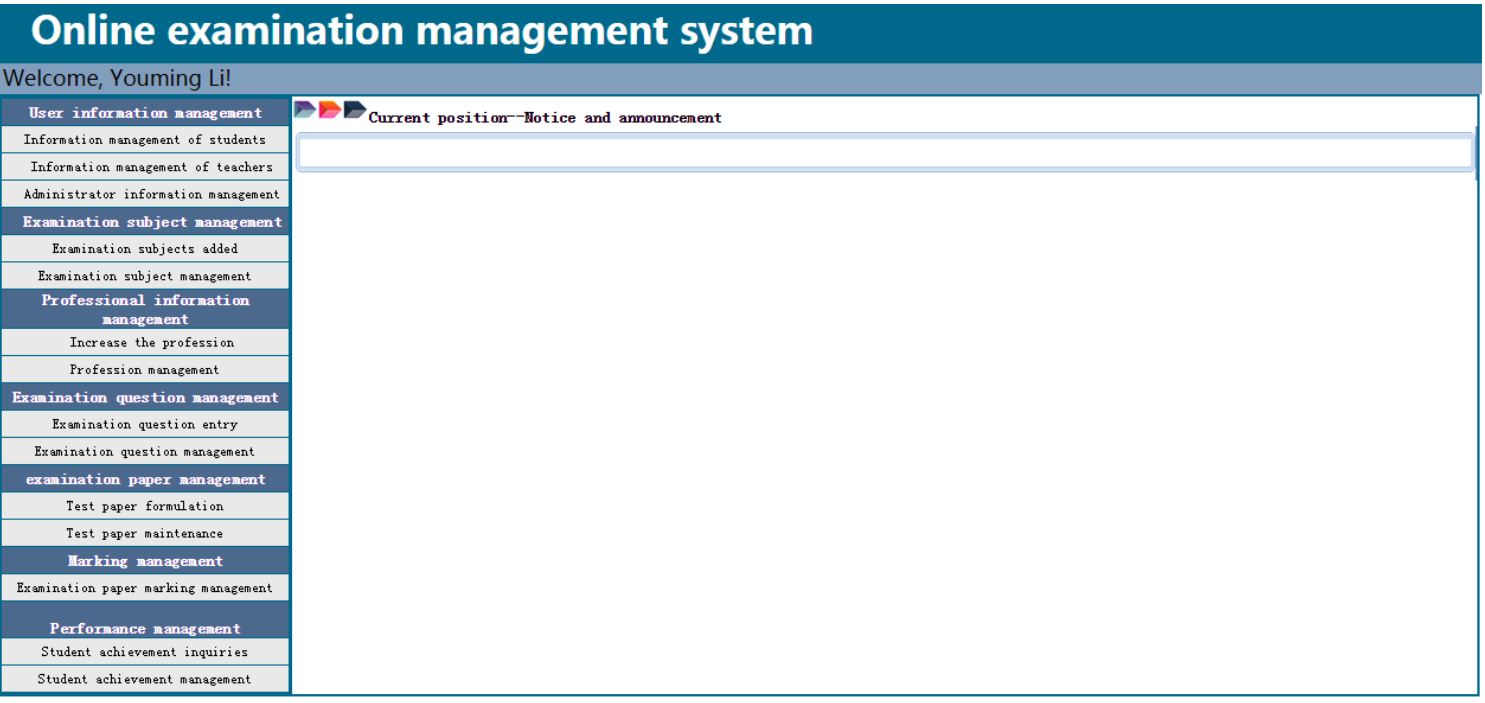

Figure 3 Backstage management interface

\subsection{Performance management interface}

\section{Online examination management system}

Welcome, Youming Li!

User information manag ement

Information management of students

Information management of teachers

Administrator information management

Examination subject nanag enent

Examination subjects added

Examination subject management

Professional infornation

Increase the professi

Profession nanagenent

Exanination question managenent

Examination question entry

Examination question manazement

exanination paper nanagement

Test paper formulation

Test paper maintenance

flarking nanagenent

Examination paper marking manazement

Performance nanazent

Student achievent ingiries

Student achievement inquiries

Student achievement management

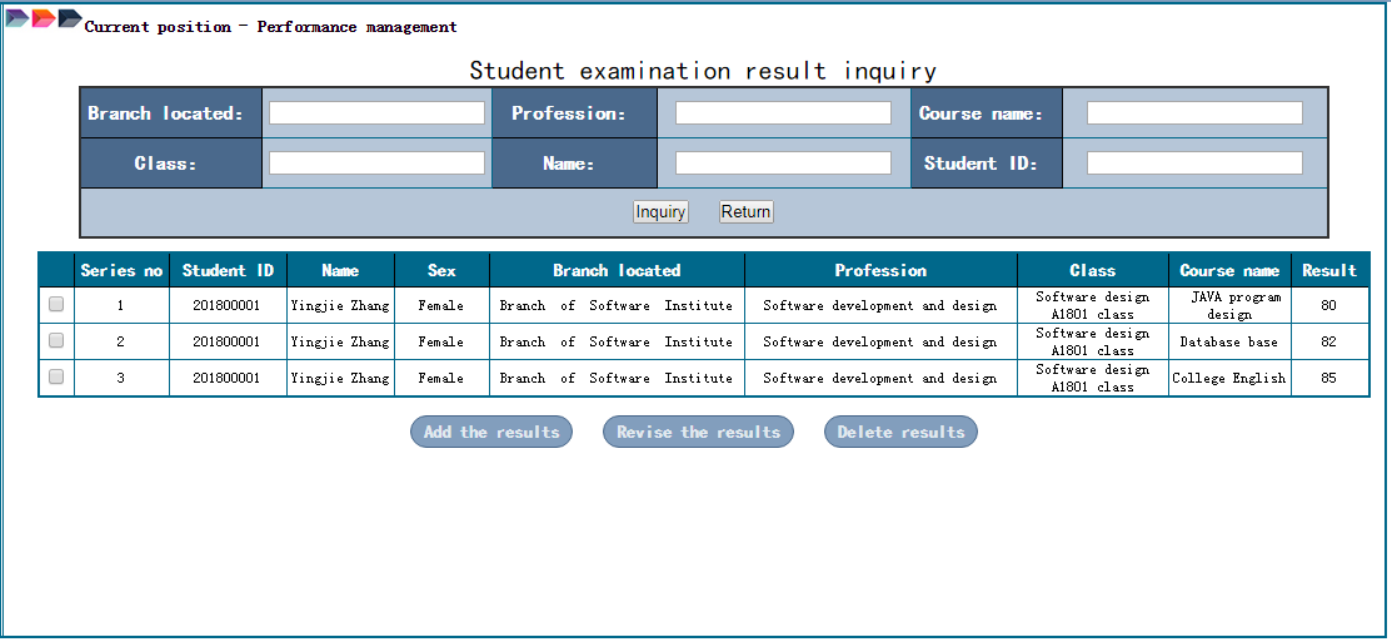

Figure 4 Performance management interface 


\subsection{Interface of examination and answer}

\section{The final examination of JAVA program design}

Total score of examination papers: 100 Test time: 2 hours

Student ID: 201800001, Name: Yingjie Zhang! Welcome to online examination system. Good luck!

- Single choice (the total of 20 items, each item of 1 points, a total of 20 points)

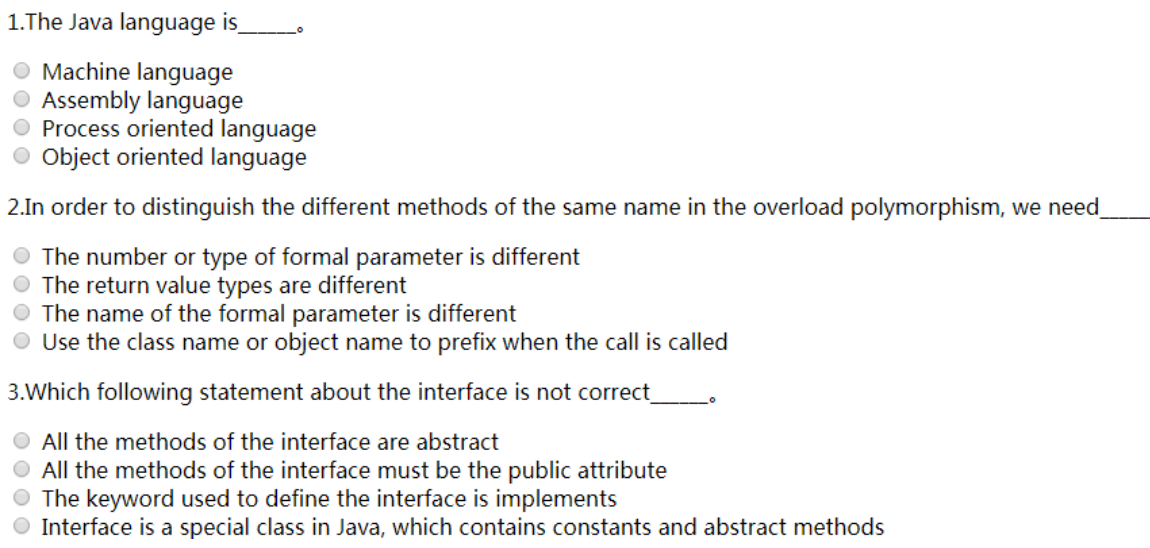

Figure 5 interface of examination and answer

\section{Conclusion}

The online examinee system is a powerful application system based on the computer network. The design of this system is based on the popular B/S mode and C\# language, the purpose of this system is to explore a test pattern based on the campus network. Through this new mode, the long and complicated process of traditional examination mode has been changed. Making full use of the convenience environment of the campus network to carry out examination to students anytime and anywhere, greatly simplifies the traditional examination process, reduces the work intensity of the teaching management departments and teachers, and improves the work efficiency and standardization level.

\section{References}

[1] Xin Cao, Hong He, Yana Zhang, Li Han. Discussion on the design and implementation of online examination system in colleges and universities [J]. Knowledge and encouragement, 2016, (16)

[2] Min Tan, Qiang Fan, Yu Tong. Research and design of online examination system, [J]. information channel, 2017 (9).

[3] Xiaobo Ma. C\# program development practical tutorial [M]. Beijing: Tsinghua University press, 2013

[4] Yongxiang Bai, Yuan Yuan. The design and implementation of online examination system based on C\# [J]. computer knowledge and technology, 2016 (27).

[5] Wenfan Wang, Lili Shi. Design and implementation of online examination system based on ASP.NET. [J]. Technology economy market, 2017 (7). 